\title{
Growth limitation of Staphylococcus epidermidis in biofilms contributes to rifampin tolerance
}

\author{
* Corresponding author: \\ DrP. S. Stewart \\ Center for Biofilm Engineering \\ Montana State University \\ 366 EPS Building \\ Bozeman \\ MT 59717-3980, USA \\ T 14069942890 \\ F 14069946098 \\ E phil_s@erc.montana.edu
}

Center for Biofilm Engineering and Department of Chemical Engineering, Montana State University-Bozeman, Bozeman, MT 59717-3980, USA

\section{Z. Zheng and P. S. Stewart*}

\begin{abstract}
Tolerance of Staphylococcus epidermidis in biofilms to killing by rifampin was correlated with limitation of bacterial growth in the biofilm state. Intact biofilm experienced a $0.62 \mathrm{log}$ reduction when treated with $0.1 \mu \mathrm{g}$ rifampin $/ \mathrm{ml}$ for $4 \mathrm{~h}$ whereas the same treatment of exponential-phase planktonic cells produced a log reduction of 4.48. Stationary-phase planktonic cells were nearly as tolerant as intact biofilm cells, experiencing a $1.11 \mathrm{log}$ reduction. Biofilm bacteria grew at only $10 \%$ of the maximum rate at which they grew on the same medium in planktonic culture. Killing was localized near the surface of the biofilm adjacent to the nutrient source, as revealed by staining with a respiratory dye. Increased nutrient concentration during antibiotic treatment enhanced killing of biofilm cells. Changing the oxygen tension in the gas phase above the biofilm during antibiotic treatment barely affected killing. It was hypothesized that the biofilm harbors significant numbers of stationary-phase-like cells in the nutrient-limited depths of the biofilm, and that these inactive cells are the ones that survive antibiotic challenge.
\end{abstract}

\section{INTRODUCTION}

Staphylococci that adhere to implanted medical devices or damaged tissue can cause persistent infections such as those related to catheters, prosthetic heart valves, vascular grafts and osteomylitis (Costerton et al., 1999). These bacteria evade antimicrobial chemotherapy by growing in biofilms in which they are protected. While the reduced antibiotic susceptibility of bacteria in biofilms is widely recognized, the reasons for this protection remain unclear (Stewart \& Costerton, 2001).

We have reported that rifampin penetrates biofilms formed by Staphylococcus epidermidis but fails to effectively kill the bacteria (Zheng \& Stewart, 2002). This result corroborates other studies of antibiotic penetration in staphylococcal biofilms which concluded that poor antibiotic penetration was not an adequate explanation for reduced susceptibility in the biofilm state (Dunne et al., 1993; Darouiche et al., 1994; Yasuda et al., 1994). The purpose of the work reported in this article was to test the hypothesis that slow killing of S. epidermidis biofilms by rifampin was due to nutrient limitation and slow or no growth of biofilm bacteria (Xu et al., 2000).

The likely role of slow growth of biofilm cells in contributing to their protection from antimicrobial agents is a longstanding hypothesis (Brown et al., 1988). Several investigations support this mechanism for staphylococcal biofilms in particular. Venables and coworkers showed that Staphylococcus aureus undergo physiological changes in the early stages of biofilm formation (Williams et al., 1999). These changes included smaller cell size, lower respiratory activity per cell and lower membrane potential in the attached population. This same group also reported that adherent $S$. aureus grew more slowly than free-floating bacteria (Williams et al., 1997). Hodgson et al. (1995) measured slow growth of bacteria in $S$. aureus biofilms. Bacteria in the biofilm grew at an average specific growth rate of $0.06 \mathrm{~h}^{-1}$ whereas freefloating cells grew at a rate of $0.7 \mathrm{~h}^{-1}$ in the same medium. Duguid et al. (1992a,b) demonstrated growth rate-modulated sensitivity of $S$. epidermidis to tobramycin and ciprofloxacin; the more slowly the cells grew, the less susceptible they were to killing. Prior work therefore supports the ideas that staphylococcal biofilms harbor cells with reduced metabolic activity and that less active cells are less susceptible.

\section{MATERIALS AND METHODS}

\section{Biofilm formation and antibiotic susceptibility}

Biofilms of S. epidermidis ATCC 35984 were grown in continuous flow reactors by dripping one-tenth strength tryptic soy broth (TSB: Difco, Detroit, MI) over inclined stainless steel coupons in a covered chamber. Medium was applied dropwise at the top of a $7.8 \mathrm{~cm} \times 1.2 \mathrm{~cm}$ coupon 
and flowed down the long dimension of the coupon tilted at an angle of $10^{\circ}$ to the horizontal. Medium drained from the reactor to a waste reservoir. The fluid residence time in the chamber was a few minutes or less. The chamber was covered, with ports on the lid that allowed for the introduction of gases other than air. The reactor system has been described in detail elsewhere (Stewart et al., 2001). The medium flow rate was $50 \mathrm{ml} / \mathrm{h}$. All work was carried out at $37^{\circ} \mathrm{C}$.

The susceptibility of biofilm bacteria to antibiotics was measured using $48 \mathrm{~h}$ biofilms. The flow over the biofilm was switched from one-tenth strength TSB to onetenth strength TSB containing either $0.1 \mu \mathrm{g}$ rifampin $/ \mathrm{ml}$ or $2.5 \mu \mathrm{g}$ ciprofloxacin $/ \mathrm{ml}$. These concentrations of antibiotics were approximately 10 times the minimum inhibitory concentrations for planktonic cells. In some experiments, the strength of TSB during the antibiotic treatment period was varied or the gas phase above the biofilm was changed from air to oxygen or nitrogen. After exposure to the antibiotic for periods ranging from 4 to $48 \mathrm{~h}$, biofilm was scraped from the sample slide into $50 \mathrm{ml}$ of buffered water. Aggregates, dispersed by brief processing with a tissue homogenizer, were enumerated for viable cell numbers by serial dilution and plating on tryptic soy agar (TSA). The degree of killing was calculated as a log reduction using an untreated control as the reference cell count.

\section{Planktonic antibiotic susceptibility}

Planktonic bacteria were grown in shake flasks in onetenth strength TSB at $37^{\circ} \mathrm{C}$. The susceptibility of planktonic bacteria was measured by adding antibiotic to a subculture that was diluted in one-tenth strength TSB to an optical density 0.02 ( $1 \mathrm{~cm}$ path length) at $600 \mathrm{~nm}$ immediately prior to treatment. In some experiments the inocula were derived from exponential phase cultures $(6 \mathrm{~h})$ and in others the inocula came from stationary phase cultures $(30 \mathrm{~h})$. Rifampin was then added at $0.1 \mu \mathrm{g} / \mathrm{ml}$ and the culture incubated with shaking at $37^{\circ} \mathrm{C}$. Surviving bacteria were enumerated by serial dilution and plating on TSA.

\section{Growth rates}

The growth rate of bacteria in suspension cultures was determined by linear regression of natural log cell number versus time data; the slope of this line gave the growth rate. The average growth rate of biofilm bacteria was calculated from a material balance on cells. The average growth rate is equal to the effluent cell concentration multiplied by the volumetric flow rate of medium and divided by the total number of biofilm cells on the sample coupon.

\section{Respiratory activity staining and microscopy}

Biofilms were stained with $0.16 \%$ 5-cyano-2,3-ditolyltetrazolium chloride (CTC) for $2 \mathrm{~h}$ at $37^{\circ} \mathrm{C}$ (Huang et al., 1995), cryoembedded (Yu et al., 1994), frozen sectioned,

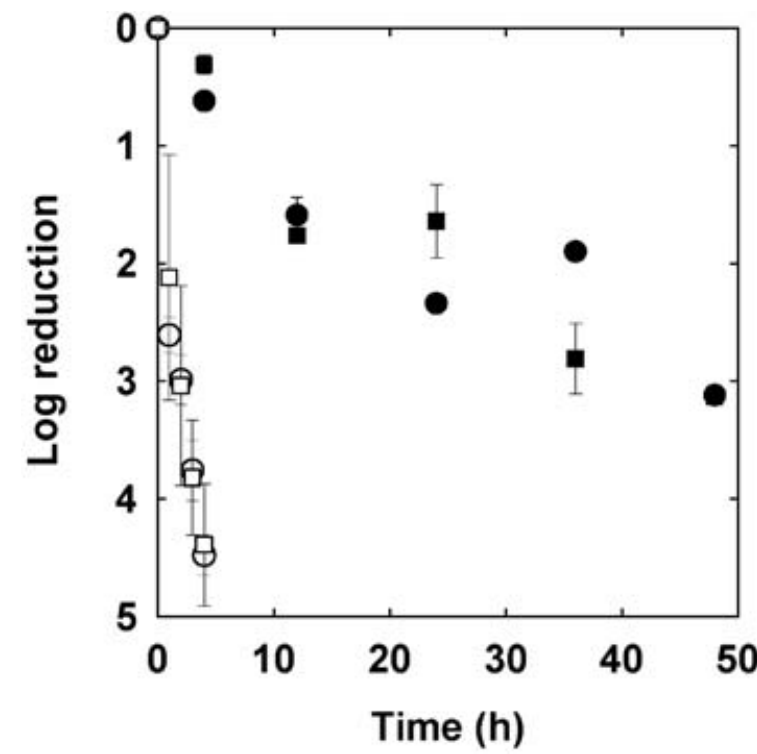

Fig. 1: Comparison of susceptibility of $S$. epidermidis exposed to $0.1 \mu \mathrm{g}$ rifampin $/ \mathrm{ml}$ (circles) or $2.5 \mu \mathrm{g}$ ciprofloxacin $/ \mathrm{ml}$ (squares) in the planktonic (open symbols) and biofilm (filled symbols) states. The treatment time was $4 \mathrm{~h}$. Error bars indicate the standard error of the mean.

and counterstained with the DNA stain 4',6-diamidino2-phenylindole (DAPI), applied at $10 \mu \mathrm{g} / \mathrm{ml}$ for $3 \mathrm{~min}$. Specimens were examined with a Nikon E800 microscope using epifluorescence illumination.

\section{RESULTS}

\section{Staphyloccocus epidermidis biofilms are protected from killing by rifampin and ciprofloxacin}

Free-floating bacteria from an exponential-phase growing culture were more susceptible than biofilm bacteria to killing by rifampin (Fig. 1). The untreated control biofilm at the time of initiation of treatment $(48 \mathrm{~h})$ was covered with a lush biofilm with a viable areal cell density of $8.94 \pm 0.12 \log _{10} \mathrm{cfu} / \mathrm{cm}^{2}$. After $4 \mathrm{~h}$ of treatment, the log reduction in viable cell numbers for the treated biofilm was $0.62 \pm 0.13$. The $\log$ reduction of growing planktonic bacteria in response to the same treatment was $4.48 \pm 0.34$. This difference was statistically significant $(P=0.002)$. Staphyloccocus epidermidis biofilms were also less susceptible to killing by $2.5 \mu \mathrm{g}$ ciprofloxacin/ml by comparison with growing planktonic bacteria (Fig. 1). With this antibiotic, the log reduction after $4 \mathrm{~h}$ treatment was only $0.32 \pm 0.08$ for biofilms as compared with $4.39 \pm 0.52$ for planktonic cells.

\section{Stationary-phase planktonic cells are protected from killing by rifampin}

Bacteria taken from a stationary-phase planktonic culture were less susceptible to killing by rifampin than were 
Table 1: Comparison of growth rates and susceptibilities of exponential-phase planktonic, stationary phase planktonic, resuspended biofilm, and intact biofilm bacteria. Killing was measured in response to $4 \mathrm{~h}$ rifampin treatment

\begin{tabular}{|c|c|c|c|}
\hline Source of bacteria & $\begin{array}{l}\text { Log reduction } \\
\text { untreated control }\end{array}$ & $\begin{array}{l}\text { Log reduction } \\
\text { treated }\end{array}$ & $\begin{array}{l}\text { Growth rate } \\
\left(\mathrm{h}^{-1}\right)\end{array}$ \\
\hline Exponential phase planktonic & $-1.84 \pm 0.54$ & $4.48 \pm 0.34$ & $0.82 \pm 0.34$ \\
\hline Stationary phase planktonic & $-0.29 \pm 0.11$ & $1.11 \pm 0.05$ & $0.15 \pm 0.06$ \\
\hline Resuspended biofilm & $-0.93 \pm 0.17$ & $1.53 \pm 0.06$ & $0.51 \pm 0.03$ \\
\hline Intact biofilm & 0 & $0.62 \pm 0.13$ & $0.08 \pm 0.02$ \\
\hline
\end{tabular}

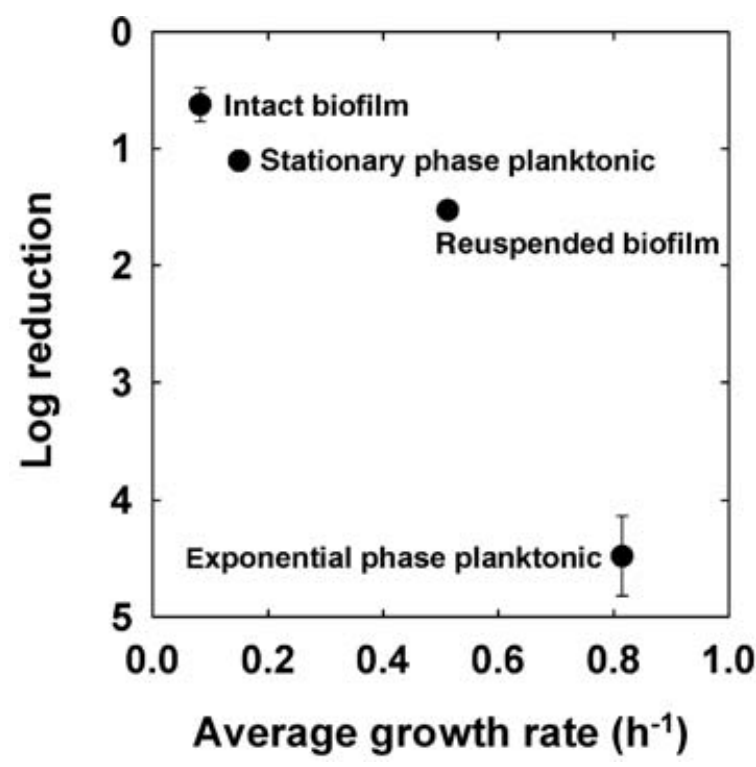

Fig. 2: Staphyloccocus epidermidis killing by $0.1 \mu \mathrm{g}$ rifampin $/ \mathrm{ml}$ as a function of growth rate. The treatment time was $4 \mathrm{~h}$. Error bars indicate the standard error of the mean.

bacteria taken from an actively growing culture. The log reduction of bacteria taken from a stationary-phase culture was $1.11 \pm 0.05$, only about one-quarter of the kill measured when inocula were taken from growing cultures (Table 1). When bacteria were dispersed from biofilms by scraping and homogenization, resuspended in one-tenth strength TSB, and treated with rifampin, the degree of killing was similar to that measured for stationary phase planktonic cells. The log reduction after $4 \mathrm{~h}$ treatment in this case was $1.53 \pm 0.06$. These results show that the growth status of the bacteria prior to, and at the time of, the antibiotic challenge is an important factor in determining susceptibility.

There was a rough correlation between average growth rate and rifampin sensitivity (Fig. 2). The more slowly bacteria grew, the less susceptible they were to killing by rifampin. Bacteria in the biofilm mode of growth were least susceptible, and also grew most slowly. The average specific growth rate of biofilm bacteria was only $0.08 \pm 0.02 \mathrm{~h}^{-1}$. For comparison, the specific growth rate of bacteria in a rapidly growing planktonic culture was an order of magnitude larger, $0.82 \pm 0.34 \mathrm{~h}^{-1}$. The trend of decreasing susceptibility with decreasing growth rate was statistically significant $(P=0.04)$. Having established that the

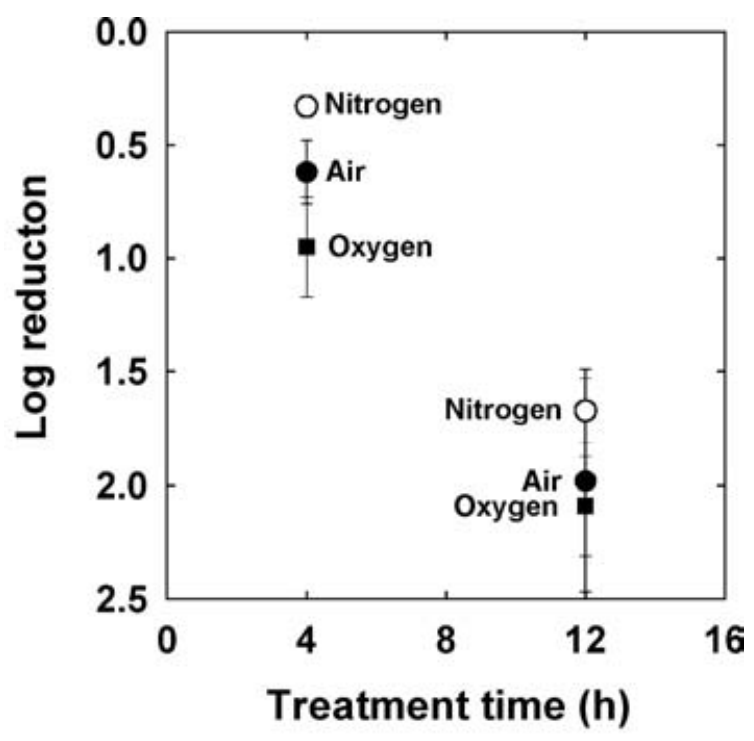

Fig. 3: S. epidermidis biofilm susceptibility to $0.1 \mu \mathrm{g}$ rifampin $/ \mathrm{ml}$ as a function of oxygen availability. Error bars indicate the standard error of the mean.

growth status of the bacteria influences their antibiotic susceptibility, we hypothesized that biofilm susceptibility could be modulated by changing the level of oxygen or nutrients provided to the bacteria during antibiotic treatment. Experiments were therefore conducted in which biofilms were grown by the standard procedure and then challenged with $0.1 \mu \mathrm{g}$ rifampin $/ \mathrm{ml}$ under differing conditions of oxygen and nutrient provision. When the gas phase above the biofilm was changed from air to nitrogen or pure oxygen, little change in the extent of killing by rifampin was detected (Fig. 3). While these experiments supported a trend in increased killing as the oxygen tension increased, the differences were not statistically significantly different at the 95\% confidence level in most cases. The statistical test used for this and other comparisons was a simple twosided t-test. We conclude that oxygen availability was not a major factor in controlling the susceptibility of S. epidermidis biofilms under these conditions.

On the other hand, when the nutrient strength was changed during antibiotic treatment, a clear effect on the susceptibility of biofilm bacteria was evident (Fig. 4). Higher nutrient concentration during antibiotic treatment led to greater killing, while more dilute nutrients led to less killing. The trend of antibiotic killing increasing 


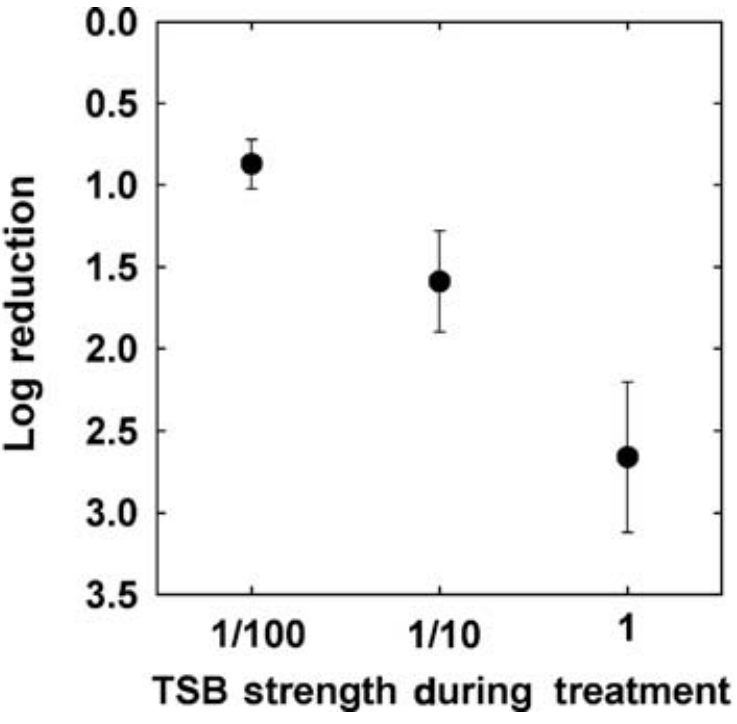

Fig. 4: Staphyloccocus epidermidis biofilm susceptibility to $0.1 \mu \mathrm{g}$ rifampin $/ \mathrm{ml}$ as a function of nutrient concentration. The treatment time was $12 \mathrm{~h}$. Error bars indicate the standard error of the mean.

with nutrient strength was statistically significant $(P=$ 0.01 ). These experiments were conducted by growing the biofilms in the standard way, in one-tenth strength TSB. Only during the $12 \mathrm{~h}$ period of antibiotic treatment was the TSB strength changed. The log reduction was calculated in comparison to an untreated (no antibiotic) control that underwent the same upward shift in nutrient composition. These results suggest that nutrient availability may modulate rifampin susceptibility in these S. epidermidis biofilms.

If nutrient diffusion into the biofilm were important, then one would expect to see greater metabolic activity and greater antibiotic efficacy near the surface of the biofilm and reduced metabolic activity and diminished antibiotic action in the depths of the biofilm. As a test of this possibility, S. epidermidis biofilms were stained with the respiratory dye CTC. This stain deposits fluorescent red crystals at the site of respiratory activity. The use of the DNA intercalating dye DAPI stains all cells blue, independently of their metabolic state. Distinct patterns of respiratory activity, as indicated by CTC staining, were observed in control and antibiotic-treated biofilms (Fig. 5).

In the untreated control, an intense layer of red CTCformazan formed at the biofilm-bulk fluid interface (Fig. 5A). The CTC staining was less intense near the substratum. DAPI counterstaining of the same section revealed a uniform blue intensity throughout the depth of the biofilm (Fig. 5A). From image analysis of DAPIstained sections, the thickness of the untreated control biofilm was $636 \pm 64 \mu \mathrm{m}$. In a biofilm treated with $0.1 \mu \mathrm{g}$ rifampin/ml for $4 \mathrm{~h}$, respiratory activity was suppressed near the biofilm-bulk fluid interface and was more intense throughout the remainder of the biofilm (Fig. 5B). DAPI staining again indicated a fairly uniform distribution of biomass (Fig. 5B). Image analysis of the treated biofilm
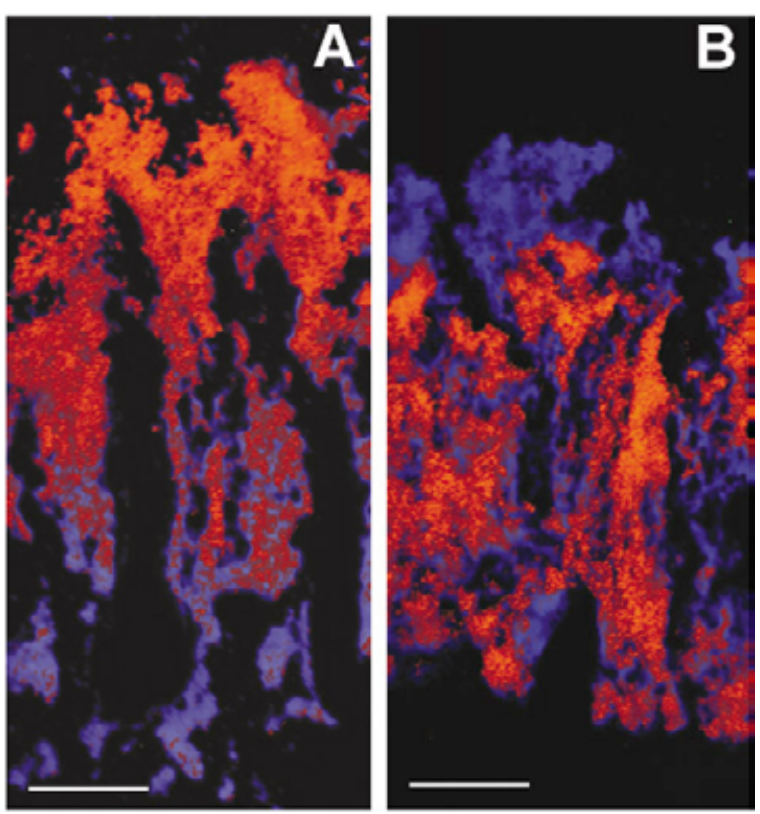

Fig. 5: Patterns of respiratory activity (red) and biomass distribution (blue) in untreated (A) and rifampin-treated (B) S. epidermidis biofilms. The treatment time was $12 \mathrm{~h}$. Respiratory activity was visualized by CTC staining and biomass was revealed by staining DNA with DAPI. The substratum is at the bottom and the biofilm-nutrient interface at the top in both panels. The scale bar indicates $100 \mu \mathrm{m}$.

returned an average biofilm thickness of $466 \pm 13 \mu \mathrm{m}$ and indicated that the zone of reduced respiratory activity near the biofilm surface was $91 \pm 13 \mu \mathrm{m}$ in dimension. These results suggest that rifampin exerted its greatest activity near the surface of the biofilm, where nutrients are presumably most available.

\section{DISCUSSION}

The results of this study support the hypothesis that nutrient limitation resulting in slow or no growth of bacteria in $S$. epidermidis biofilm is responsible for tolerance to rifampin (Zimmerli et al., 1994; Schierholz et al., 1998). Evidence consistent with this mechanism includes: a general correlation, for both planktonic and biofilm bacteria, between growth rate and susceptibility; demonstration that the extent of killing depends on nutrient availability during antibiotic treatment; and the observation that antibiotic action is localized at the biofilm-nutrient interface. The lack of influence of oxygen tension on biofilm susceptibility measured in this study is consistent with the data of Norden \& Shaffer (1983), who reported that minimum inhibitory concentrations and minimum bactericidal concentrations of rifampin challenging $S$. aureus were identical under aerobic and anaerobic conditions.

Because most antibiotics target macromolecular synthesis, it should not be surprising that slowly growing or non-growing cells are less susceptible to killing. Rifampin binds to DNA-dependent RNA polymerase and inhibits initiation of RNA synthesis. Ciprofloxacin binds 
to gyrase, interferes with proper supercoiling of DNA and eventually halts replication. In a bacterial cell in which there is neither DNA replication nor transcription occurring, these antibiotics cannot be expected to have much effect. Antibiotics might still kill slow-growing cells over time, but against dormant or stationary-phase cells these agents might have little efficacy even during prolonged periods of exposure.

It is important to recognize that the growth of bacteria in a biofilm is likely to vary with spatial location in the biofilm (Xu et al., 2000). Consider, by way of illustration, a biofilm in which cells grow, on average, at half the rate of their planktonic counterparts. This population might actually comprise two halves: one that is growing rapidly, at the full planktonic rate; and the other that is in stationary phase and not growing at all. Characterization of biofilm populations by average growth rates, to which we have also resorted in this article, may not be an adequate way of characterizing the growth status of a biofilm population. We suggest that it is those cells that are not growing at all, which are of the greatest importance in understanding antibiotic tolerance in biofilms. More work is needed to prove that biofilms harbor significant numbers of non-growing, yet viable, cells.

Given the likelihood that biofilms contain stationaryphase-like cells, batch cultures are arguably more appropriate than continuous culture for planktonic comparisons with biofilms. A cell in a biofilm probably experiences a history that is more like that in a batch culture. When the biofilm is thin, cells may be in exponential phase. As the biofilm gets thicker, those cells near the surface of the biofilm may continue to grow rapidly. But those cells that become more deeply embedded in the biofilm will experience diminishing nutrients and eventual complete depletion of nutrients. This progression is similar to a batch culture that enters stationary phase. Biofilm cells are unlikely to have the chemostat experience of a steady, precise nutrient level that maintains a certain growth rate over a long period.

\section{ACKNOWLEDGEMENT}

Peg Dirckx helped to prepare the color figure.

\section{REFERENCES}

Brown, M. R. W., Allison, D. G. \& Gilbert, P. (1988) Resistance of bacterial biofilms to antibiotics: a growth-rate related effect? Journal of Antimicrobial Chemotherapy 22, 777-783

Costerton, J. W., Stewart, P. S. \& Greenberg, E. P. (1999) Bacterial biofilms: a common cause of persistent infections. Science 284, 1318-1322

Darouiche, R. O., Dhir, A., Miller, A. J., Landon, G. C., Raad, I. I. \& Musher, D. M. (1994) Vancomycin penetration into biofilm covering infected prostheses and effect on bacteria. Journal of Infectious Diseases 170, 720-723

Duguid, I. G., Evans, E., Brown, M. R. W. \& Gilbert, P. (1992a) Effect of biofilm culture upon the susceptibility of Staphylococcus epidermidis to tobramycin. Journal of Antimicrobial Chemotherapy 30, 803-810

Duguid, I. G., Evans, E., Brown, M. R. W. \& Gilbert, P. (1992b) Growth-rate-independent killing by ciprofloxacin of biofilm-derived Staphylococcus epidermidis: evidence for cell-cycle dependency. Journal of Antimicrobial Chemotherapy 30, 791-802

Dunne, W. M. Jr, Mason, E. O. Jr \& Kaplan, S. L. (1993) Diffusion of rifampin and vancomycin through a Staphylococcus epidermidis biofilm. Antimicrobial Agents and Chemotherapy 37, 2522-2526

Hodgson, A. E., Nelson, S. M., Brown, M. R. W. \& Gilbert, P. (1995) A simple in vitro model for growth control of bacterial biofilms. Journal of Applied Bacteriology 79, 87-93

Huang, C.-T., Yu, F. P., McFeters, G. A. \& Stewart, P. S. (1995) Nonuniform spatial patterns of respiratory activity within biofilms during disinfection. Applied Environmental Microbiology 61, 2252-2256

Nordern, C. W. \& Shaffer, M. (1983) Treatment of experimental chronic osteomyelitis due to Staphylococcus aureus with vancomycin and rifampin. Journal of Infectious Diseases 147, 352-357

Schierholz, J. M., Beuth, J. \& Pulverer, G. (1998) Killing effects of antibiotics and two-fold antimicrobial combinations on proliferating and non-growing staphylococci. Zentralblatt für Bakteriologie 288, 527-539

Stewart, P. S. \& Costerton, J. W. (2001) Antibiotic resistance of bacteria in biofilms. Lancet 358, 135-138

Stewart, P. S., Rayner, J., Roe, F. \& Rees, W. M. (2001) Biofilm penetration and disinfection efficacy of alkaline hypochlorite and chlorosulfamates. Journal of Applied Microbiology 91, $525-532$

Williams, I., Venables, W. A., Lloyd, D., Paul, F. \& Critchley, I. (1997) The effects of adherence to silicone surfaces on antibiotic susceptibility in Staphylococcus aureus. Microbiology 143, 2407-2413

Williams, I., Paul, F., Lloyd, D., Jepras, R., Critchely, I., Newman, M., Warrack, J., Giokarini, T., Hayes, A. J., Randerson, P. F. \& Venables, W. A. (1999) Flow cytometry and other techniques show that Staphylococcus aureus undergoes significant physiological changes in the early stages of surface-attached culture. Microbiology 145, 1325-1333

Xu, K. D., McFeters, G. A. \& Stewart, P. S. (2000) Biofilm resistance to antimicrobial agents. Microbiology 146, 547-549

Yasuda, H., Ajiki, Y., Koga, T. \& Yokota, T. (1994) Interaction between clarithromycin and biofilms formed by Staphylococcus epidermidis. Antimicrobial Agents and Chemotherapy 38, $138-141$

Yu, F. P., Callis, G. M., Stewart, P. S., Griebe, T. \& McFeters, G. A. (1994) Cryosectioning of biofilms for microscopic examination. Biofouling 8, 85-91

Zheng, Z. \& Stewart, P. S. (2002). Penetration of rifampin through Staphylococcus epidermidis biofilms. Antimicrobial Agents and Chemotherapy 46, 900-903

Zimmerli, W., Frei, R., Widmer, A. F. \& Rajacic, Z. (1994) Microbiological tests to predict treatment outcome in experimental device-related infections due to Staphylococcus aureus. Journal of Antimicrobial Chemotherapy 33, 959-967 